\section{Sex an Weihnachten}

\section{Zum weltweiten Paarungsverhalten geschlechtsreifer Festefeierer}

\section{Spitzer, UIm}

Neun Monate nach Weihnachten - im September - steigt die Geburtenrate in den meisten „westlichen“ Ländern der Nordhalbkugel der Erde an. Dieses Faktum mag zunächst wenig verwundern, handelt es sich bei Weihnachten doch um das Fest der Liebe! - Aber warum nehmen die Leute das denn gleich so wörtlich? - Dies wird sich mancher Leser fragen, denn hierzulande ist Weihnachten doch eher das Fest der Familie, der kleinen Kinder mit den leuchtenden Augen unter dem Christbaum, des Schenkens und Beschenkt-werdens und nicht zuletzt des (allzu) vielen Essens. Rein assoziativ betrachtet, so möchte man meinen, schießt einem zu „Weihnachten“ eher nicht als erste Assoziation "Sex“ durch den Kopf, liegt also „Sex“ nicht im unmittelbaren Assoziationshof von „Weihnachten“. - Oder etwa doch?

Liegt "Sex" also nicht im unmittelbaren Assoziationshof von "Weihnachten"?

Früher hatte man neben den Geburtenraten nicht viel weitere Möglichkeiten, um herauszubekommen, was sich in den Köpfen bzw. den Schlafzimmern der Menschen im jahreszeitlichen Wechsel so abspielt. Einen ersten Hinweis jedoch lieferten die Verkaufszahlen von Kondomen, die einer britischen Studie (9) zufolge in der Weihnachtswoche nahezu doppelt so hoch lagen wie im Jahresdurchschnitt ( Abb. 1).

Wie gut, dass es heute das Internet und Suchmaschinen gibt, mit deren Hilfe man den Gedanken, Wünschen und Verhaltensweisen einer sehr großen Zahl von Men-

\section{Nervenheilkunde 2018; 37: 861-863}

Korrespondenzadresse

Prof. Dr. Dr. Manfred Spitzer, Universitätsklinikum UIm Klinik für Psychiatrie und Psychotherapie III Leimgrubenweg 12, $89075 \mathrm{Ulm}$ schen auf die Schliche kommen kann. Insbesondere der seit dem Jahr 2004 verfügbare Online-Dienst Google Trends des Unternehmens Google ist hier sehr hilfreich: Hier werden Informationen darüber bereitstellt, welche Suchbegriffe von Nutzern der Suchmaschine Google wie oft eingegeben wurden. Weltweite Bekanntheit erreichte beispielsweise die Analyse von mit Grippeerkrankungen in Verbindung stehenden Suchbegriffen zur Vorhersage der Grippe-Ausbreitung in der Bevölkerung (4). Im Vergleich zum US-amerikanischen Pendant des deutschen Robert-Koch-Instituts, des Center for Disease Prevention and Control (CDPC), sind diese Daten aus der "Google-Epidemiologie“ ein bis zwei Wochen früher verfügbar und können damit einen erheblichen praktischen Nutzen haben ${ }^{1}$.

Eine bereits im Jahr 2013 erschienene Studie der US-Amerikaner Patrick und Charlotte Markey zur Häufigkeit sexbezogener Google-Suchanfragen in den USA ergab denn auch eine deutliche Steigerung in einem halbjährigen Rhythmus mit Spitzen im Sommer und um die Jahreswende (6). Dieses Muster wurde sowohl für Suchbegriffe im Umkreis von „Pornografie“, „Prostitution“ und „Partnerschaft" gefunden. Sie leiten daraus ab, dass es um einen allgemeinen Trend im Hinblick auf das sexuelle Verlangen der Menschen geht und weniger um spezifische einzelne Suchbegriffe. Da Pornografie und Prostitution vor allem von Männern gesucht werden, ist die Tatsache von Bedeutung, dass es praktisch keine Unterschiede zwischen dem zeitlichen Verlauf der Häufigkeit dieser Suchbegriffe und dem Suchbegriff "Partnerschaft" (bezogen sich die Suchwörter vor allem auf - von beiden Geschlechtern genutzte Partnervermittlungs-Institutionen) gab so die Argumentation der Autoren.

Nun könnte es sein, dass dieses Ergebnis nur für die USA zutrifft. Und es ist weiter- hin nicht klar, ob es durch biologische oder durch kulturelle Faktoren bedingt ist. Um diesen Fragen nachzugehen, untersuchten Informatiker der Indiana University in Bloomington, USA, zusammen mit Kollegen aus Portugal und den Niederlanden die wöchentlichen Häufigkeiten von Anfragen bei Google zum Suchwort „Sex" in weltweit 129 Ländern im Zeitraum von 2004 bis 2014 (10).

Die Auswahl der Länder erfolgte nach dem einfachen praktischen Kriterium, dass es genügend auswertbare Daten (Zeitreihen mit wöchentlichen relativen Häufigkeiten der Sex-Suchanfrage, bezogen auf alle Suchanfragen) in Google-Trends über mindestens 4 Jahre hinweg gab, um länderbezogen auswerten zu können. Hierzu wurden die Länder nach ihrer geografischen Lage (nördliche versus südliche Halbkugel) als auch nach ihrer Religion (bei jeweils mehr als 50\% Christen oder Muslimen in der Gesamtbevölkerung ${ }^{2}$ ) in „christliche“, „islamische“ und „andere“ eingeteilt.

Wie bereits in der auf die USA begrenzten Studie von Markey und Markey (6) zeigte sich auch bei dieser globalen Betrachtung, dass in der Weihnachtswoche die meisten Google-Suchanfragen zu "Sex“ erfolgen. Ein Vergleich mit der Woche um

\footnotetext{
1 Wie so oft bei digitalen Neuerungen erscheinen die Dinge nach dem ersten Hype im weiteren Verlauf dann längst nicht so rosig wie beim ersten „Hit“. Der erste Massive Online Open Course (MOOG) der Stanford University war ein Riesenerfolg, aber danach zeigte sich, dass die Erfolgsrate dieser Kurse bei jämmerlichen 2-8\% liegt. Ganz ähnlich erging es der Google-Epidemiologie: Weitere Untersuchungen ergaben, dass sich keineswegs alle Grippeepidemien adäquat in Google-Trends abbildeten, gab es doch in der Folge sowohl „übersehene" als auch „unterschätzte“ Grippeepidemien (7). Am besten erscheint - ebenfalls wie so oft - die Kombination der traditionellen mit den neuen Methoden (8).

2 Der Teufel (sic!) steckt wie immer im Detail, wie das folgende Zitat aus dem Methodenteil zeigt: „Out of the countries identified as Christian, eleven have a majority that follow either the Russian or Serbian Orthodox Churches (namely: Belarus, Bosnia and Herzegovina, Bulgaria, Georgia, Macedonia, Moldova, Montenegro, Serbia, Slovenia, Russia and Ukraine). In ten of these countries (Bulgaria being the exception), Christmas is celebrated in early January (of the Gregorian Calendar) and they could have been labeled as Other for the proposes of this analysis" (10, S. e7).
} 


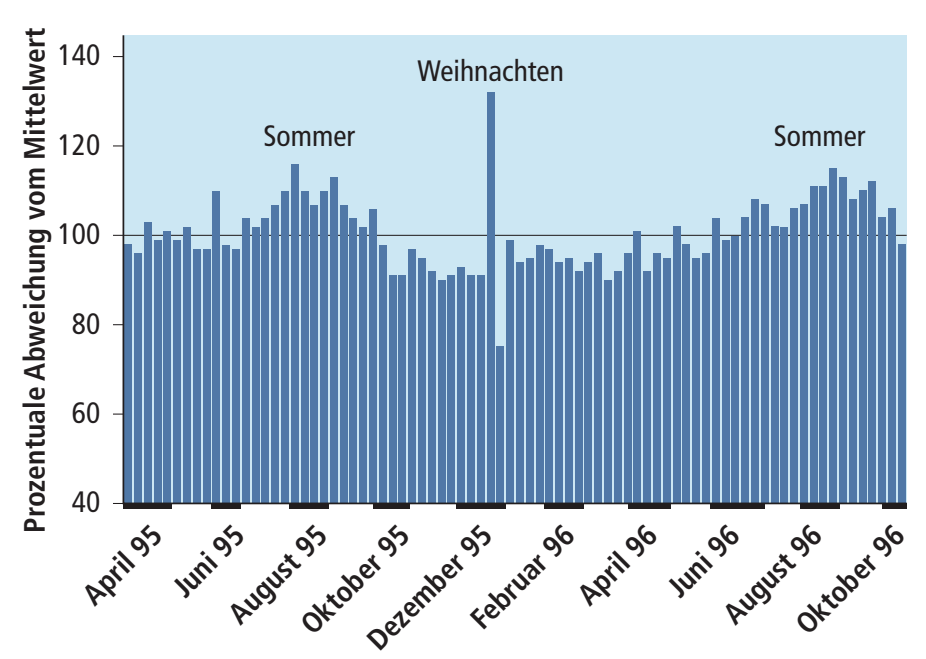

Abb. 1 Kondomverkauf pro Woche in britischen Geschäften vom April 1995 bis September 1996 (nach 9).

Thanksgiving in den USA und mit der Osterwoche in Frankreich ergab, dass dies nicht an der Tatsache, dass es Ferien gab, liegen konnte und die Leute einfach mehr Zeit hatten. Denn an diesen genannten Feiertagen ließ sich kein deutlich erhöhtes Interesse an Sex beobachten. Man bedenke zudem, dass die Häufigkeiten ja auf alle Suchanfragen bezogen (normiert) sind und damit die einfache Erklärung „die Leute haben mehr Zeit und verbringen diese im Internet" entfällt.

Dass die Google-Suchanfragen tatsächliches Verhalten abbilden, zeigt sich spätestens dann, wenn man daran, sie mit den Geburtenraten in den jeweiligen Ländern 9 Monate später in Beziehung setzt. Soweit dies möglich war, wurde dies getan und es wurde der bereits bekannte Effekt der Häufung der Geburten im September gefunden.

Kürzere Tage, kältere Temperaturen und weniger Nahrung um die Wintersonnenwende wurden als Hinweis für einen Einfluss biologischer Faktoren auf die Libido gewertet.

Man dachte für lange Zeit, dass die jahreszeitlichen Schwankungen der menschlichen Reproduktion vor allem natürlich bedingt sind: Kürzere Tage, kältere Temperaturen und weniger Nahrung um die Wintersonnenwende wurden als Hinweis für einen Einfluss biologischer Faktoren auf die Libido gewertet $(1,2,5)$.

Diese Sicht der Dinge werden durch die Studie von Wood und Mitarbeitern in Frage gestellt: Auf der südlichen Halbkugel sind die Ergebnisse genau so wie auf der nördlichen, d. h. Sonneneinstrahlung bzw. Kälte können den Effekt nicht erklären. Die Tatsache, dass Weihnachten überall am gleichen Tag gefeiert wird (also auf der Nordhalbkugel im Winter und auf der Südhalbkugel im Sommer), deutet also klar auf eine kulturelle Verursachung hin.

Untermauert wird dies durch den Befund, dass in muslimischen Ländern entsprechende Google-Suchanfragen um das Ende des Fastenmonats Ramadan (d. h. um das Fest des Fastenbrechens) ihre Spitzenwerte erreichen (und die Geburtenraten entsprechend 9 Monate später ${ }^{3}$ ). Da sich dieses Fest jedes Jahr um 10 Tage verschiebt, lässt sich auch mit dieser Beobachtung der jahreszeitliche Wandel der Lebensbedingungen als Ursache des Effekts ausschließen. Während des Fastens hingegen ist in muslimischen Ländern ein deutlicher Rückgang der sexbezogenen GoogleSuchanfragen zu verzeichnen. Die Christen wiederum scheinen ihre Fastenzeit weit weniger ernst zu nehmen, denn bei ihnen findet sich kein entsprechender Rückgang.

Klassifiziert man die einzelnen Länder nach dem Spitzenwert der Sex-Suchanfragen um Weihnachten oder um das Fest des Fastenbrechens (Eid-al-Fitr), erhält man ei- ne Karte ( $\triangleright$ Abb. 2), die kulturelle Unterschiede wiedergibt. Die Halbkugel, in der ein Land geografisch liegt, spielt dagegen keine Rolle.

Von den als christlich eingestuften Ländern zeigen $80 \%$ einen signifikanten Anstieg der sexbezogenen Google-Suchanfragen über Weihnachten.

Von den 80 als christlich eingestuften Ländern zeigen $80 \%$ einen signifikanten Anstieg der sexbezogenen Google-Suchanfragen über Weihnachten. Bei den 30 Muslimischen Ländern war in 77\% ein entsprechender signifikanter Anstieg um das Fest des Fastenbrechens herum zu verzeichnen.

Den Autoren zufolge ist der Zusammenhang zwischen beiden Festen mit erhöhtem sexuellen Interesse und entsprechendem Verhalten keineswegs intuitiv nachvollziehbar. Sie gehen daher möglichen Gründen nach und untersuchten Schwankungen der im Kurznachrichtendienst Twitter mitgeteilten Emotionen in sieben Ländern, in denen hierzu genügend Daten (mindestens 10000 Twitter-Nachrichten pro Woche) vorlagen: Australien, Argentinien, Brasilien, Chile, Indonesien, die Türkei und die USA. Der Zeitraum reichte vom September 2010 bis zum Februar 2014. Man verwendete ein Lexikon mit gut tausend Wörtern, die Affekte beschreiben und sich nach den Dimensionen „Erregung“, „Dominanz“ und "Valenz" ordnen ließen".

Die Autoren fanden in jedem untersuchten Land, dass die Anzahl der sexbezogenen Google-Anfragen mit einer erhöhten positiven Valenz und einer erniedrigten Dominanz einhergingen. In ihren eigenen Worten: „thus the happier but less in-control the population mood is, the more sexsearches tend to increase in every country" (10, S. e4). Interessanterweise traf dies so-

3 Dies gilt für die christlichen Länder. Für die muslimischen Länder führen die Autoren an, dass dort keine verlässlichen Daten zum Datum der Geburten vorliegen.

4 Es handelt sich hierbei im Grunde um die drei Dimensionen - „stark-schwach“, „aktiv-passiv“ und "gut-schlecht“, die schon von Osgood und Tannenbaum in den 1950er-Jahren mittels der Methodik des semantischen Differenzials beschrieben wurden. 


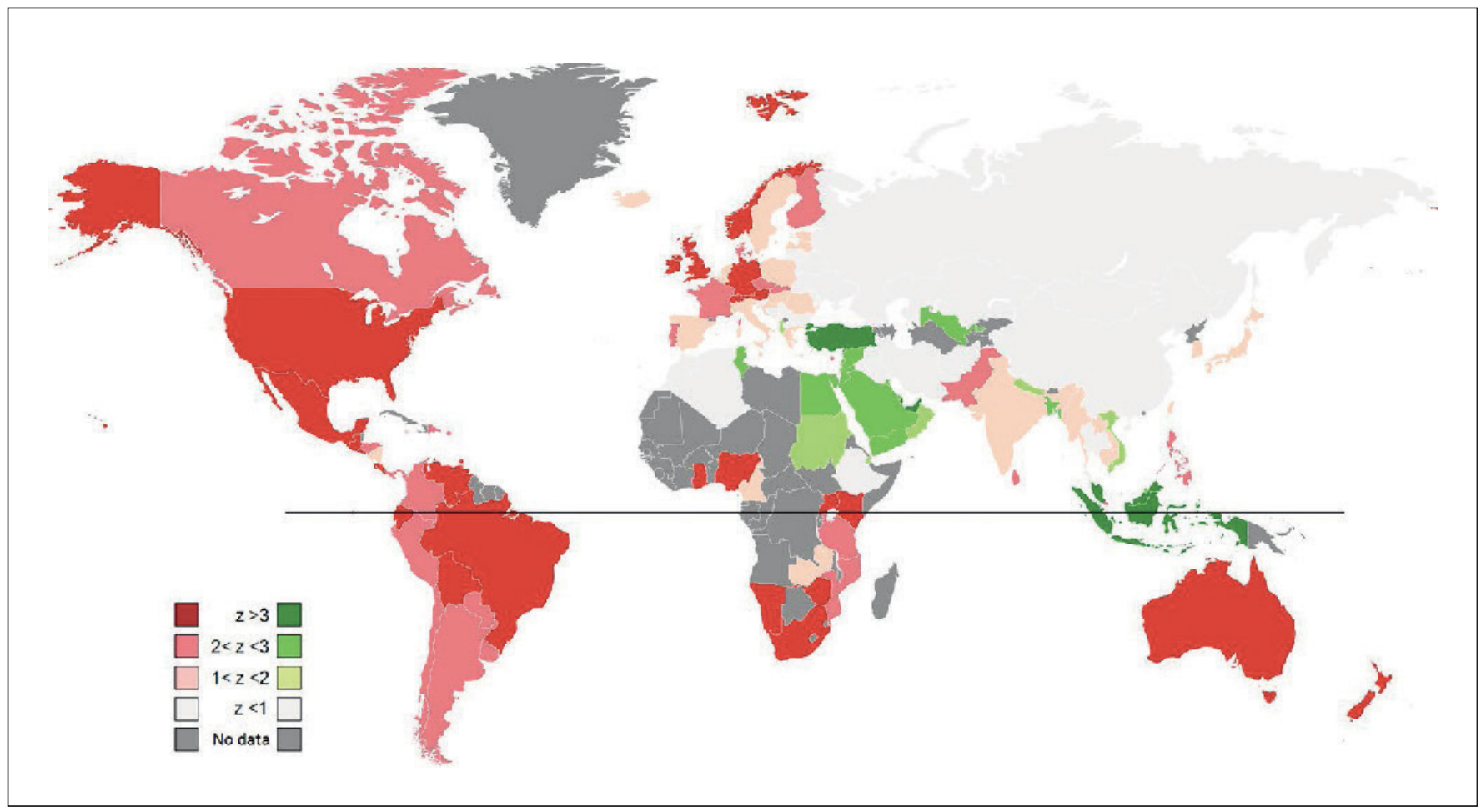

Abb. 2 Länder mit Spitzenwerten für die Google-Suchanfrage nach "Sex" in der Woche um Weihnachten (rot) bzw. der Woche um das Fastenbrechen (grün). Je dunkler der Farbwert, desto höher der Spitzenwert im Vergleich zum Jahresmittel (z-transformierter Wert der Spitze durch die Farbsättigung kodiert). Für in Hellgrau markierte Länder gibt es keinen signifikanten Spitzenwert, für in Dunkelgrau markierte Länder liegen keine Google-Trends-Daten vor (aus 10, korrigierte Version vom März 2018).

wohl auf Weihnachten in christlichen Ländern als auch auf das Fest des Fastenbrechens in muslimischen Ländern zu. Der Zusammenhang zwischen Stimmung und Sex scheint mithin bei allen Menschen ähnlich zu sein, unabhängig von der Überformung unserer Lebensgewohnheiten durch unterschiedliche Religionen.

Wer also geglaubt hat, dass man an Weihnachten gerne der Kälte und der Dunkelheit wegen kuschelt (und sich der Rest dann eben ergibt), der liegt falsch, denn auch in Südamerika, Südafrika oder Australien ergibt es sich, in brütender Hitze oder zumindest lauen Abenden. Nicht Dunkelheit und Kälte, sondern Glück, Ruhe und Sicherheit sind die Faktoren, die weltweit neun Monate nach dem großen Familienfest für mehr Nachkommen sor- gen. Und das scheint allen Menschen gemein zu sein.

\section{Literatur}

1. Bronson FH. Seasonal variation in human reproduction: environmental factors. Quarterly Review of Biology 1995; 70(2): 141-164.

2. Cummings DR. Human birth seasonality and sunshine. American Journal of Human Biology 2010; 22: 316-324.

3. Dodds PS, Harris KD, Kloumann IM, Bliss CA, Danforth CM. Temporal Patterns of Happiness and Information in a Global Social Network: Hedonometrics and Twitter. PLoS ONE 2011; 6(12): e26752.

4. Ginsberg J, Mohebbi MH, Patel RS, Brammer L, Smolinski MS, Brilliant L. Detecting influenza epidemics using search engine query data. Nature 2009; 457: 1012-1014.

5. Macdowall W, Wellings K, Stephenson J, Glasier A. Summer nights: A review of the evidence of sea- sonal variations in sexual health indicators among young people. Health Education 2007; 108: 40-53.

6. Markey PM, Markey CN. Seasonal variation in internet keyword searches: A proxy assessment of sex mating behaviors. Arch Sex Behav 2013; 42: 515-521.

7. Olson DR, Konty KJ, Paladini M, Viboud C, Simonsen L. Reassessing Google Flu Trends data for detection of seasonal and pandemic influenza: A comparative epidemiological study at three geographic scales. PLoS Comput Biol 2013; 9(10): e1003256.

8. Wagner M, Lampos V, Cox IJ, Pebody R. The added value of online user-generated content in traditional methods for influenza surveillance. Scientific Reports 2018; 8: 13963.

9. Wellings K, Macdowall W, Catchpole M, Goodrich J. Seasonal variations in sexual activity and their implications for sexual health promotion. J R Soc Med 1999; 92: 60-64.

10. Wood IB, Varela PL, Bollen J, Rocha LM, Gonçalves-Sá J. Human sexual cycles are driven by culture and match collective moods. Scientific Reports 2017; 7: 17973 . 